J. Appl. Numer. Optim. 1 (2019), No. 3, pp. 277-291

Available online at http://jano.biemdas.com

https://doi.org/10.23952/jano.1.2019.3.07

\title{
KARUSH-KUHN-TUCKER OPTIMALITY CONDITIONS AND DUALITY FOR SET OPTIMIZATION PROBLEMS WITH MIXED CONSTRAINTS
}

\author{
L.T. TUNG ${ }^{1}$, T.T. $\mathrm{KHAI}^{2}$, P.T. $\mathrm{HUNG}^{3}$, P.L.B. $\mathrm{NGOC}^{3, *}$ \\ ${ }^{1}$ Department of Mathematics, College of Natural Sciences, Can Tho University, Can Tho, Vietnam \\ ${ }^{2}$ Center for Training and Enterprise Cooperation, Tra Vinh University, Tra Vinh, Vietnam \\ ${ }^{3}$ Faculty of Pedagogy and Faculty of Social Sciences \& Humanities, Kien Giang University, Kien Giang, Vietnam
}

\begin{abstract}
In this paper, we consider set optimization problems with mixed constraints. We first investigate necessary and sufficient Karush-Kuhn-Tucker optimality conditions for strict minimal solutions. Then, we formulate types of Mond-Weir and Wolfe dual problems and explore duality relations under convexity assumptions. Some examples are provided to illustrate our results.
\end{abstract}

Keywords. Set optimization problems with mixed constraints; Strict minimal solutions; Karush-Kuhn-Tucker optimality conditions; Mond-Weir duality; Wolfe duality.

2010 Mathematics Subject Classification. 90C46, 90C34, 90C70.

\section{INTRODUCTION}

Set-valued optimization problems play an important role in optimization theory because many models in practice can be described via set-valued optimization problems; see the books $[4,12,22]$ for more details. There are two main types of approaches to define solution concepts of set-valued optimization problems: the vector approach and the set approach. The former is the usual one, the latter arises naturally in the real-world application in socio-economic; see the papers $[16,17,23]$, the survey [9] and the references therein. In the vector approach, many types of generalized derivatives were employed to establish the optimality conditions for set-valued optimization problems in two directions: the dual spaces and the primal spaces. For some recent results in this direction, see the books $[12,22]$, the papers $[2,3,7,10,13,19,20,24]$ and the references therein. In the set approach, the optimality conditions for some types of solutions of set optimization problems were established in terms of the directional derivatives of the continuous selections in [1]. The paper [14] investigated the necessary optimality conditions in terms of contingent derivatives and Mordukhovich coderivatives for optimal solutions of set optimization problems with respect to the possibly and lower set less order relation. By using Studniarski derivatives, the necessary and sufficient optimality conditions were derived in [15] for optimal solutions of set optimization problems with respect to the lower set less order relation. To the best of our knowledge, there is no paper dealing with establishing optimality conditions for the

\footnotetext{
${ }^{*}$ Corresponding author.

E-mail addresses: 1ttung@ctu.edu.vn (L.T. Tung), khai@tvu.edu.vn (T.T. Khai), pthung@vnkgu.edu.vn (P.T. Hung), plbngoc@vnkgu.edu.vn (P.L.B.Ngoc).

Received July 9, 2019; Accepted September 30, 2019.
}

(C)2019 Journal of Applied and Numerical Optimization 
set optimization problems with mixed constraints. Moreover, the Mond-Weir and Wolfe dual problems of set optimizations with mixed constraints also have not been investigated yet.

Motivated by the above observations, in this paper, we establish Karush-Kuhn-Tucker optimality conditions and discuss the duality problems for the set optimization problems with mixed constraints. The paper is organized as follows. Section 2 recalls basic concepts and some preliminaries. The Karush-Kuhn-Tucker necessary and sufficient optimality conditions for strict minimal solutions are established in Section 3. Section 4 is devoted to exploring Mond-Weir and Wolfe dual problems of the set optimization problems with mixed constraints. Some examples are provided to illustrate our results.

\section{PRELIMinaries}

In this paper, let $X, Y, Z$ and $W$ be Banach spaces, $C \subseteq Y, D \subseteq Z$ be pointed closed convex cones with nonempty interior. Denote $X^{*}$ the topological dual space of $X$. The notion $B_{X}$ represents the open unit ball of $X$ and $B_{X}(x, \delta)$ stands for the open ball with center $x \in X$ and radius $\delta$. For a given set $A \subseteq X, \operatorname{int} A, \operatorname{cl} A, \partial A$, cone $A$ denote its interior, closure, boundary and the cone $\{\lambda a \mid \lambda \geq 0, a \in A\}$ of $A$, respectively (resp). A point $\bar{y}$ is said to be a weak efficient point of $B \subset Y$ with respect to (w.r.t.) $C$, denoted by $\bar{y} \in \operatorname{WMin}_{C} B$, iff $(B-\bar{y}) \cap(-\operatorname{int} C)=\emptyset$. A subset $B \subset Y$ has the weakly $C$-minimal property if for all $y \in B$ there exists $\bar{y} \in \mathrm{WMin}_{C} B$ such that $\bar{y}-y \in-\operatorname{int} C \cup\{0\}$, i.e., $B-\bar{y} \subset \operatorname{int} C \cup\{0\}$. For the cones $C \subseteq Y$ and $D \subseteq Z$,

$$
\begin{aligned}
& C^{+}:=\left\{y^{*} \in Y^{*} \mid\left\langle y^{*}, c\right\rangle \geq 0, \forall c \in C\right\}, \\
& D^{+}:=\left\{d^{*} \in Z^{*} \mid\left\langle d^{*}, z\right\rangle \geq 0, \forall z \in D\right\}
\end{aligned}
$$

are positive polar cones of $C, D$, resp. A convex set $B \subset Y$ is called a base for $C$ if $0 \notin \operatorname{cl} B$ and $C=\left\{t b \mid t \in \mathbb{R}_{+}, b \in B\right\}$.

Definition 2.1. $[4,12]$ Let $A$ be a nonempty subset of $X$.

(i) The contingent (or Bouligand) cone of $A$ at $\bar{x} \in \operatorname{cl} A$ is

$$
T(A, \bar{x}):=\left\{x \in X \mid \exists t_{n} \downarrow 0, \exists x_{n} \rightarrow x, \forall n \in \mathbb{N}, \bar{x}+t_{n} x_{n} \in A\right\} .
$$

(ii) The adjacent cone of $A$ at $\bar{x} \in \operatorname{cl} A$ is

$$
T^{b}(A, \bar{x}):=\left\{x \in X \mid \forall t_{n} \downarrow 0, \exists x_{n} \rightarrow x, \forall k \in \mathbb{N}, \bar{x}+t_{n} x_{n} \in A\right\} .
$$

(iii) The interior tangent cone of $A$ at $\bar{x}$ is

$$
I T(A, \bar{x}):=\left\{x \in X \mid \forall t_{n} \downarrow 0, \forall x_{n} \rightarrow x, \forall n \text { large }, \bar{x}+t_{n} x_{n} \in A\right\} .
$$

Remark 2.1. The following properties can be checked directly.

(i) $\operatorname{IT}(A, \bar{x}) \subset T^{b}(A, \bar{x}) \subset T(A, \bar{x})$.

(ii) $0 \in T(A, \bar{x})$ and $0 \in I T(A, \bar{x})$ if $\bar{x} \in \operatorname{int} A$.

(iii) $[4,12]$ If $A$ is a convex set then $T^{b}(A, \bar{x})=T(A, \bar{x})=\operatorname{clcone}(A-\bar{x})$.

(iv) $[11,12]$ If $A$ is a convex set then $I T(A, \bar{x})=\operatorname{intcone}(A-\bar{x})=\operatorname{cone}(\operatorname{int} A-\bar{x})$. 
For a given set-valued map $P: X \rightrightarrows Y$, the domain, graph and epigraph of $P$ are defined respectively by

$$
\begin{aligned}
\operatorname{dom} P & :=\{x \in X: P(x) \neq \emptyset\}, \\
\operatorname{gr} P & :=\{(x, y) \in X \times Y: y \in P(x)\}, \\
\text { epi } P & :=\{(x, y) \in X \times Y: y \in P(x)+C\} .
\end{aligned}
$$

The so-called profile mapping of $P$ is $P_{+}$defined by $P_{+}(x)=P(x)+C . P$ is $C$-convex if its epigraph is convex, i.e., for all $x, x^{\prime} \in X$ and $\lambda \in[0,1]$,

$$
\lambda P(x)+(1-\lambda) P\left(x^{\prime}\right) \subset P\left(\lambda x+(1-\lambda) x^{\prime}\right)+C .
$$

Definition 2.2. [8] Let $P: X \rightrightarrows Y$ be a set-valued map, $(\bar{x}, \bar{y}) \in \operatorname{gr} P$ and $u \in X$. $P$ is said to be directionally metrically subregular at $(\bar{x}, \bar{y})$ in direction $u$ w.r.t. $S \subset X$ iff there are $\alpha \geq 0$ and $\gamma>0$ such that, for all $t \in] 0, \gamma\left[\right.$ and $v \in B_{X}(u, \gamma)$ with $\bar{x}+t v \in S$,

$$
d\left(\bar{x}+t v, P^{-1}(\bar{y}) \cap S\right) \leq \alpha d(\bar{y}, P(\bar{x}+t v)) .
$$

Definition 2.3. [4] Let $P: X \rightrightarrows Y$ and $(\bar{x}, \bar{y}) \in \operatorname{gr} P$.

(i) The set-valued map $P$ is said to be Aubin at $(\bar{x}, \bar{y})$ if there exist neighborhoods $U$ of $\bar{x}$ and $V$ of $\bar{y}$, and $L>0$ such that

$$
P(x) \cap V \subset P\left(x^{\prime}\right)+L\left\|x-x^{\prime}\right\| \mathrm{cl} B_{Y}, \forall x, x^{\prime} \in U .
$$

(ii) The set-valued map $P$ is said to be $C$-Aubin at $(\bar{x}, \bar{y})$ if there exist neighborhoods $U$ of $\bar{x}$ and $V$ of $\bar{y}$, and $L>0$ such that

$$
P(x) \cap V \subset P\left(x^{\prime}\right)+C+L\left\|x-x^{\prime}\right\| \operatorname{cl} B_{Y}, \forall x, x^{\prime} \in U .
$$

Definition 2.4. Let $P: X \rightrightarrows Y$ be a set-valued map and $(\bar{x}, \bar{y}) \in \operatorname{gr} P$.

(i) [4] The contingent derivative $D P(\bar{x}, \bar{y})$ of $P$ at $(\bar{x}, \bar{y})$ is

$$
D P(\bar{x}, \bar{y})(u):=\left\{v \in Y \mid \exists t_{n} \downarrow 0, \exists\left(u_{n}, v_{n}\right) \rightarrow(u, v), \forall n \in \mathbb{N}, \bar{y}+t_{n} v_{n} \in P\left(\bar{x}+t_{n} u_{n}\right)\right\} .
$$

(ii) [4] The adjacent derivative $D^{b} P(\bar{x}, \bar{y})$ of $P$ at $(\bar{x}, \bar{y})$ is

$$
D^{b} P(\bar{x}, \bar{y})(u):=\left\{v \in Y \mid \forall t_{n} \downarrow 0, \exists\left(u_{n}, v_{n}\right) \rightarrow(u, v), \forall n \in \mathbb{N}, \bar{y}+t_{n} v_{n} \in P\left(\bar{x}+t_{n} u_{n}\right)\right\} .
$$

(iii) [7] The lower (Dini) derivative $D^{l} P(\bar{x}, \bar{y})$ of $P$ at $(\bar{x}, \bar{y})$ is

$$
D^{l} P(\bar{x}, \bar{y})(u):=\left\{v \in Y \mid \forall t_{n} \downarrow 0, \forall u_{n} \rightarrow u, \exists v_{n} \rightarrow v, \forall n \in \mathbb{N}, \bar{y}+t_{n} v_{n} \in P\left(\bar{x}+t_{n} u_{n}\right)\right\} .
$$

(iv) [7] The Dubovitskii-Miljutin derivative $D^{D M} P(\bar{x}, \bar{y})$ of $P$ at $(\bar{x}, \bar{y})$ is

$$
D^{D M} P(\bar{x}, \bar{y})(u):=\left\{v \in Y \mid \forall t_{n} \downarrow 0, \forall\left(u_{n}, v_{n}\right) \rightarrow(u, v), \forall n \text { large }, \bar{y}+t_{n} v_{n} \in P\left(\bar{x}+t_{n} u_{n}\right)\right\} .
$$

Remark 2.2. Let $P: X \rightrightarrows Y$ be a set-valued map, $(\bar{x}, \bar{y}) \in \operatorname{gr} P$ and $u \in X$.

(i) $D^{D M} P(\bar{x}, \bar{y})(u) \subset D^{l} P(\bar{x}, \bar{y})(u) \subset D^{b} P(\bar{x}, \bar{y})(u) \subset D P(\bar{x}, \bar{y})(u)$.

(ii) [4] If $P$ is convex then $D^{b} P(\bar{x}, \bar{y})(u)=D P(\bar{x}, \bar{y})(u)$.

(iii) If $P$ is $C$-convex then $D^{b} P_{+}(\bar{x}, \bar{y})(u)=D P_{+}(\bar{x}, \bar{y})(u)$.

(iv) [7] If $P$ is Aubin at $(\bar{x}, \bar{y})$ then $D^{l} P(\bar{x}, \bar{y})(u)=D^{b} P(\bar{x}, \bar{y})(u)$.

(v) If $P$ is $C$-Aubin at $(\bar{x}, \bar{y})$ then $D^{l} P_{+}(\bar{x}, \bar{y})(u)=D^{b} P_{+}(\bar{x}, \bar{y})(u)$. 
Lemma 2.1. [6] Let $P: X \rightrightarrows Y,(\bar{x}, \bar{y}) \in \operatorname{gr} P$, and $P$ be $C$-convex. Then, for all $x \in X$,

$$
P(x)-\bar{y} \subseteq D P_{+}(\bar{x}, \bar{y})(x-\bar{x}) .
$$

Let $A$ and $B$ be nonempty subsets of $Y$, denoted by $A, B \in \mathscr{P}(Y)$. Recall some set relations $[16,17]$ based on cone ordering $C$ as follows:

(i) $A \preceq_{C}^{l} B \Leftrightarrow A+C \supseteq B$,

(ii) $A \prec_{C}^{l} B \Leftrightarrow A+$ int $C \supseteq B$.

\section{KARUSH-KuHN-TUCKER Optimality CONDITIONS}

In the sequel, let $F: X \rightrightarrows Y, G: X \rightrightarrows Z, H: X \rightrightarrows W$ be three set-valued maps. We consider the following set optimization problem with mixed constraints:

$$
(P): \preceq_{C}^{l}-\min _{x \in X}\{F(x) \mid G(x) \cap(-D) \neq \emptyset, 0 \in H(x)\} .
$$

Denote $\Omega:=\{x \in X \mid G(x) \cap(-D) \neq \emptyset, 0 \in H(x)\}$ the set of feasible points of $(P)$. A point $\bar{x} \in \Omega$ is a strict minimal solution [1] with respect to $\prec_{C}^{l}$ of $(\mathrm{P})$, denoted by $\bar{x} \in \operatorname{Min}\left(P, \prec_{C}^{l}\right)$, if for any $x \in \Omega$, one has $F(x) \nprec_{C}^{l} F(\bar{x})$.

Lemma 3.1. [18] Let $\bar{x} \in X$ and $\bar{y} \in F(\bar{x})$. Suppose that $(F-\bar{y}, G, H)(X)+(\operatorname{int} C \times D \times\{0\})$ is a convex set and $\operatorname{int} H(X) \neq \emptyset$, where $(F-\bar{y})(x):=F(x)-\bar{y}$. If the system

$$
(F(x)-\bar{y}) \cap-\operatorname{int} C \neq \emptyset, G(x) \cap-D \neq \emptyset, 0 \in H(x)
$$

admits no solution $x \in X$, then there exists $\left(c^{*}, d^{*}, w^{*}\right) \in\left(C^{+} \times D^{+} \times W^{*}\right) \backslash\{(0,0,0)\}$ such that

$$
\left\langle c^{*}, y-\bar{y}\right\rangle+\left\langle d^{*}, z\right\rangle+\left\langle w^{*}, w\right\rangle \geq 0,
$$

for all $x \in X$ and for all $(y, z, w) \in(F, G, H)(x)$.

For the sake of convenience, we always assume that $\operatorname{dom} D\left(F_{+}, G_{+}, H\right)(x, y, z, w)=X$ and $\operatorname{dom} D F_{+}(x, y) \cap \operatorname{dom} D G_{+}(x, z) \cap \operatorname{dom} D H(x, w)=X$ for all $x \in X, y \in F(x), z \in G(x) \cap-D$ and $w \in H(x)$.

\subsection{Necessary Optimality Conditions.}

Proposition 3.1. Let $\bar{x} \in X, \bar{y} \in F(\bar{x}), \bar{z} \in G(\bar{x}) \cap-D, 0 \in H(\bar{x})$ and $\bar{x}$ be a strict minimal solution w.r.t. $\prec_{C}^{l}$ of $(P)$. Suppose that the following conditions hold:

(i) $\mathrm{WMin}_{C} F(\bar{x})=\{\bar{y}\}, F(\bar{x})$ has the weakly $C$-minimal property,

(ii) $(F-\bar{y}, G, H)(X)+(\operatorname{int} C \times D \times\{0\})$ is a convex set and int $H(X) \neq \emptyset$.

Then, there exists $\left(c^{*}, d^{*}, w^{*}\right) \in\left(C^{+} \times D^{+} \times W^{*}\right) \backslash\{(0,0,0)\}$ such that

$$
\left\langle c^{*}, y\right\rangle+\left\langle d^{*}, z\right\rangle+\left\langle w^{*}, w\right\rangle \geq 0
$$

and

$$
\left\langle d^{*}, \bar{z}\right\rangle=0,
$$

for all $x \in X$ and for all $(y, z, w) \in D\left(F_{+}, G_{+}, H\right)(\bar{x}, \bar{y}, \bar{z}, 0)(x)$.

Moreover, $c^{*} \in C^{+} \backslash\{0\}$ if the following constraint qualification holds

(SCQ) $: 0 \in \operatorname{int} H(X)$ and there exists $\hat{x} \in X$ such that $G(\hat{x}) \cap-\operatorname{int} D \neq \emptyset$ and $0 \in H(\hat{x})$. 
Proof. We first prove that $(F(x)-\bar{y}) \cap-\operatorname{int} C=\emptyset$ for any $x \in \Omega$. Suppose to the contrary that there exists $\hat{x} \in \Omega$ such that $(F(\hat{x})-\bar{y}) \cap-\operatorname{int} C \neq \emptyset$. Then, there exists $\hat{y} \in F(\hat{x})$ satisfying $\hat{y}-\bar{y} \in-\operatorname{int} C$, or equivalently, $\bar{y} \in \hat{y}+\operatorname{int} C$. Since $\operatorname{WMin}_{C} F(\bar{x})=\{\bar{y}\}$ and $F(\bar{x})$ has weakly $C$-minimal property, one has

$$
F(\bar{x}) \subseteq \bar{y}+\operatorname{int} C \cup\{0\} \subseteq F(\hat{x})+C+\operatorname{int} C \cup\{0\} \subseteq F(\hat{x})+\operatorname{int} C,
$$

contradicting with the fact that $\bar{x}$ is a strict minimal solution w.r.t. $\prec_{C}^{l}$ of (P). Hence, for any $x \in \Omega,(F(x)-\bar{y}) \cap-\operatorname{int} C=\emptyset$. This leads that the system

$$
(F(x)-\bar{y}) \cap-\operatorname{int} C \neq \emptyset, G(x) \cap-D \neq \emptyset, 0 \in H(x)
$$

admits no solution $x \in X$. Then, we deduce from (ii) and Lemma 3.1 the existence of $\left(c^{*}, d^{*}, w^{*}\right)$ $\in C^{+} \times D^{+} \times W^{*} \backslash\{(0,0,0)\}$ such that, for all $x \in X$ and for all $(y, z, w) \in(F, G, H)(x)$,

$$
\left\langle c^{*}, y-\bar{y}\right\rangle+\left\langle d^{*}, z\right\rangle+\left\langle w^{*}, w\right\rangle \geq 0 .
$$

We justify that $\left\langle d^{*}, \bar{z}\right\rangle=0$. Indeed, by taking $x=\bar{x}, y=\bar{y} \in F(\bar{x}), z=\bar{z} \in G(\bar{x})$ and $w=0 \in H(\bar{x})$ in (3.3), we obtain that $\left\langle d^{*}, \bar{z}\right\rangle \geq 0$. On the other hand, $d^{*} \in D^{+}$and $\bar{z} \in-D$ give that $\left\langle d^{*}, \bar{z}\right\rangle \leq 0$. Thus, $\left\langle d^{*}, \bar{z}\right\rangle=0$. Since $c^{*} \in C^{+}$and $d^{*} \in D^{+}$, we derive from (3.3) that, for all $x \in X$ and for all $(y, z, w) \in(F, G, H)(x)+(C \times D \times\{0\})$,

$$
\left\langle c^{*}, y-\bar{y}\right\rangle+\left\langle d^{*}, z-\bar{z}\right\rangle+\left\langle w^{*}, w\right\rangle \geq 0 .
$$

Finally, let $x \in X$ and $(y, z, w) \in D\left(F_{+}, G_{+}, H\right)(\bar{x}, \bar{y}, \bar{z}, 0)(x)$. Then, there exist $t_{n} \downarrow 0, x_{n} \in X$ and $\left(y_{n}, z_{n}, w_{n}\right) \in(F, G, H)\left(x_{n}\right)+(C \times D \times\{0\})$ such that

$$
\left(\frac{x_{n}-\bar{x}}{t_{n}}, \frac{y_{n}-\bar{y}}{t_{n}}, \frac{z_{n}-\bar{z}}{t_{n}}, \frac{w_{n}-0}{t_{n}}\right) \rightarrow(x, y, z, w) .
$$

From (3.4), one yields

$$
\left\langle c^{*}, \frac{y_{n}-\bar{y}}{t_{n}}\right\rangle+\left\langle d^{*}, \frac{z_{n}-\bar{z}}{t_{n}}\right\rangle+\left\langle w^{*}, \frac{w_{n}-0}{t_{n}}\right\rangle \geq 0 .
$$

Letting $n \rightarrow+\infty$ in the above inequality, we get

$$
\left\langle c^{*}, y\right\rangle+\left\langle d^{*}, z\right\rangle+\left\langle w^{*}, w\right\rangle \geq 0 .
$$

Now, let (SCQ) hold. We will verify that $c^{*} \neq 0$ by contraposition. Suppose that $c^{*}=0$. Then, from (3.3), for all $(z, w) \in(G, H)(X)+D \times\{0\}$,

$$
\left\langle d^{*}, z\right\rangle+\left\langle w^{*}, w\right\rangle \geq 0 .
$$

Employing (SCQ) leads us to the existence of $\hat{z} \in G(\hat{x})$ with $-\hat{z} \in \operatorname{int} D$ and $0 \in H(\hat{x})$. Since $d^{*} \in D^{+}$and $\hat{z} \in-$ int $D$, one gets

$$
\left\langle d^{*}, \hat{z}\right\rangle<0 .
$$

If $d^{*} \neq 0$, then we deduce from $(\hat{z}, 0) \in(G, H)(\bar{x})+D \times\{0\} \subset(G, H)(X)+D \times\{0\}$ and (3.5) that

$$
\left\langle d^{*}, \hat{z}\right\rangle=\left\langle d^{*}, \hat{z}\right\rangle+\left\langle w^{*}, 0\right\rangle \geq 0
$$

contradicting with (3.6), so $d^{*}=0$. Since $0 \in \operatorname{int} H(X)$, it follows from (3.5) that there is $\delta>0$ satisfying $\left\langle w^{*}, w\right\rangle \geq 0$ for all $w \in B_{W}(0, \delta) \subseteq H(X)$, i.e., $w^{*}=0$. This is unreasonable because $\left(c^{*}, d^{*}, w^{*}\right) \neq(0,0,0)$. Hence, $c^{*} \neq 0$. 
In the following we give a result, similar to Proposition 3.1, for the case when the derivatives of objective and constraint functions are considered separately.

Proposition 3.2. Let $\bar{x} \in X, \bar{y} \in F(\bar{x}), \bar{z} \in G(\bar{x}) \cap(-D), 0 \in H(\bar{x})$ and $\bar{x}$ be a strict minimal solution w.r.t. $\prec_{C}^{l}$ of $(P)$. Suppose that the following provisos hold:

(i) $\mathrm{WMin}_{C} F(\bar{x})=\{\bar{y}\}, F(\bar{x})$ has the weakly $C$-minimal property,

(ii) $F, G, H$ are $C$-convex, $D$-convex, convex, respectively,

(iii) $F_{+}$is $C$-Aubin at $(\bar{x}, \bar{y}), G_{+}$is D-Aubin at $(\bar{x}, \bar{z})$,

(iv) $H$ is directionally metrically subregular at $(\bar{x}, 0)$ w.r.t. $X$.

Then there exists $\left(c^{*}, d^{*}, w^{*}\right) \in C^{+} \times D^{+} \times W^{*} \backslash\{(0,0,0)\}$ such that $\left\langle d^{*}, \bar{z}\right\rangle=0$ and

$$
\left\langle c^{*}, y\right\rangle+\left\langle d^{*}, z\right\rangle+\left\langle w^{*}, w\right\rangle \geq 0
$$

for every $x \in X, y \in D F_{+}(\bar{x}, \bar{y})(x), z \in D G_{+}(\bar{x}, \bar{z})(x)$ and $w \in D H(\bar{x}, 0)(x)$. Moreover, $c^{*} \neq 0$ if the following constraint qualification holds

$$
(\mathrm{KRZCQ}):\left(D G_{+}(\bar{x}, \bar{z}), D H(\bar{x}, 0)\right)(X)+\operatorname{cone}(D+\bar{z}) \times\{0\}=Z \times W .
$$

Proof. By similar arguments as in Proposition 3.1, we infer from (i) that

$$
(F(x)-\bar{y}) \cap-\operatorname{int} C=\emptyset, \forall x \in \Omega .
$$

Moreover, we derive from (ii), (iii) and Remark 2.2 that $D^{l} F_{+}(\bar{x}, \bar{y})(x)=D F_{+}(\bar{x}, \bar{y})(x)$ and $D^{l} G_{+}(\bar{x}, \bar{z})(x)=D G_{+}(\bar{x}, \bar{z})(x)$.

Firstly, we verify that, for all $x \in X$,

$$
\left(D^{l} F_{+}(\bar{x}, \bar{y})(x), D^{l} G_{+}(\bar{x}, \bar{z})(x), D H(\bar{x}, 0)(x)\right) \cap((-\operatorname{int} C) \times(-\operatorname{int} D-\bar{z}) \times\{0\})=\emptyset .
$$

Arguing by contradiction, suppose that $x \in X$ and $(y, z) \in(-\operatorname{int} C) \times(-\operatorname{int} D-\bar{z})$ such that

$$
(y, z, 0) \in\left(D^{l} F_{+}(\bar{x}, \bar{y})(x), D^{l} G_{+}(\bar{x}, \bar{z})(x), D H(\bar{x}, 0)(x)\right) .
$$

As $0 \in D H(\bar{x}, 0)(x)$, there exist $t_{n} \downarrow 0,\left(x_{n}, w_{n}\right) \rightarrow(x, 0)$ satisfying $t_{n} w_{n} \in H\left(\bar{x}+t_{n} x_{n}\right)$. We deduce from the directional metrical subregularity of $H$ the existence of $\gamma>0$ such that, for $n$ large enough,

$$
d\left(\bar{x}+t_{n} x_{n}, H^{-1}(0)\right) \leq \gamma d\left(0, H\left(\bar{x}+t_{n} x_{n}\right)\right) .
$$

Thus, there is $x_{n}^{\prime} \in H^{-1}(0)$ such that

$$
\left\|\bar{x}+t_{n} x_{n}-x_{n}^{\prime}\right\| \leq \gamma\left\|t_{n} w_{n}\right\|
$$

Setting $\bar{x}_{n}:=\frac{1}{t_{n}}\left(x_{n}^{\prime}-\bar{x}\right)$, we have $\bar{x}+t_{n} \bar{x}_{n}=x_{n}^{\prime} \in H^{-1}(0),\left\|x_{n}-\bar{x}_{n}\right\| \leq \gamma\left\|w_{n}\right\|$, and hence, $\bar{x}_{n} \rightarrow$ $x$. As $z \in D^{l} G_{+}(\bar{x}, \bar{z})(x) \cap(-\operatorname{int} D-\bar{z})$, for the above $t_{n}$ and $x_{n}$, there exist $z_{n} \rightarrow z$ fulfilling $\bar{z}+t_{n} z_{n} \in G\left(\bar{x}+t_{n} x_{n}\right)+D$. Moreover, since $z \in-\operatorname{int} D-\bar{z}, z_{n} \rightarrow z$ and $-\operatorname{int} D-\bar{z}$ is open, one has $\bar{z}+t_{n} z_{n} \in-\operatorname{int} D$ for $n$ large. Therefore, $\left(G\left(\bar{x}+t_{n} x_{n}\right)+D\right) \cap(-\operatorname{int} D) \neq \emptyset$ leads to $G\left(\bar{x}+t_{n} x_{n}\right) \cap(-D) \neq \emptyset$. This implies that $\bar{x}+t_{n} x_{n} \in \Omega$ for large $n$.

Since $y \in D^{l} F_{+}(\bar{x}, \bar{y})(x)$, for the above $t_{n}$ and $x_{n}$, there exist $y_{n} \rightarrow y$ and $c_{n} \in C$ such that $\bar{y}+t_{n} y_{n}-c_{n} \in F\left(\bar{x}+t_{n} x_{n}\right)$ for all $n \in \mathbb{N}$. As $y \in-\operatorname{int} C, y_{n} \rightarrow y$ and $-\operatorname{int} C$ is open, one has $t_{n} y_{n} \in-\mathrm{int} C$ for $n$ large enough. Hence, $t_{n} y_{n}-c_{n} \in-\operatorname{int} C-C=-\operatorname{int} C$. Thus, there exists $\bar{x}+t_{n} x_{n} \in \Omega$ such that

$$
t_{n} y_{n}-c_{n} \in\left(F\left(\bar{x}+t_{n} x_{n}\right)-\bar{y}\right) \cap(-\operatorname{int} C),
$$

contradicting with (3.7). This confirms (3.8). 
Moreover, we derive from (ii) that $\left(D F_{+}(\bar{x}, \bar{y})(x), D G_{+}(\bar{x}, \bar{z})(x), D H(\bar{x}, 0)(x)\right)$ is a convex set. Hence, it follows from (3.8) and the standard separation theorem that there exists $\left(c^{*}, d^{*}, w^{*}\right) \in$ $Y^{*} \times Z^{*} \times W^{*} \backslash(0,0,0)$ such that

$$
\left\langle c^{*}, y\right\rangle+\left\langle d^{*}, z\right\rangle+\left\langle w^{*}, w\right\rangle \geq\left\langle c^{*}, c^{\prime}\right\rangle+\left\langle d^{*}, d^{\prime}\right\rangle+\left\langle d^{*},-\bar{z}\right\rangle,
$$

for all $x \in X,(y, z, w) \in\left(D F_{+}(\bar{x}, \bar{y})(x), D G_{+}(\bar{x}, \bar{z})(x), D H(\bar{x}, 0)(x)\right), c^{\prime} \in-\operatorname{intC}$ and $d^{\prime} \in-\operatorname{int} D$. We assert by the assumptions -intC and -intD are cones that

$$
\left\langle c^{*}, y\right\rangle+\left\langle d^{*}, z\right\rangle+\left\langle w^{*}, w\right\rangle+\left\langle d^{*}, \bar{z}\right\rangle \geq\left\langle c^{*}, \varepsilon c^{\prime}\right\rangle+\left\langle d^{*}, \eta d^{\prime}\right\rangle,
$$

for all $x \in X,(y, z, w) \in\left(D F_{+}(\bar{x}, \bar{y})(x), D G_{+}(\bar{x}, \bar{z})(x), D H(\bar{x}, 0)(x)\right), c^{\prime} \in-\operatorname{intC}, d^{\prime} \in-\operatorname{int} D, \varepsilon>$ 0 and $\eta>0$. We claim that $\left\langle c^{*}, c\right\rangle \geq 0$ for all $c \in \operatorname{intC}$. If otherwise, there exists $0 \neq \bar{c} \in \operatorname{int} C$ such that $\left\langle c^{*}, \bar{c}\right\rangle<0$. Then, $0 \neq \bar{c}^{\prime}:=-\bar{c} \in-$ int $C$ and $\left\langle c^{*}, \bar{c}^{\prime}\right\rangle>0$. Hence, we deduce from (3.9) that

$$
\left\langle c^{*}, y\right\rangle+\left\langle d^{*}, z\right\rangle+\left\langle w^{*}, w\right\rangle+\left\langle d^{*}, \bar{z}\right\rangle \geq\left\langle c^{*}, \varepsilon \bar{c}^{\prime}\right\rangle+\left\langle d^{*}, \eta d^{\prime}\right\rangle,
$$

which leads to a contradiction when passing $\varepsilon \rightarrow+\infty$. Thus, $\left\langle c^{*}, c\right\rangle \geq 0$ for all $c \in \operatorname{intC}$, and consequently,

$$
\left\langle c^{*}, c\right\rangle \geq 0, \forall c \in C=\operatorname{cl} C=\operatorname{cl}(\text { intC }),
$$

i.e., $c^{*} \in C^{+}$. By similar arguments, one gets $d^{*} \in D^{+}$.

Now, we will prove that $\left\langle d^{*}, \bar{z}\right\rangle=0$. Letting $\varepsilon \rightarrow 0^{+}$and $\eta \rightarrow 0^{+}$in (3.9), we ensure that

$$
\left\langle c^{*}, y\right\rangle+\left\langle d^{*}, z\right\rangle+\left\langle w^{*}, w\right\rangle+\left\langle d^{*}, \bar{z}\right\rangle \geq 0,
$$

for all $x \in X,(y, z, w) \in\left(D F_{+}(\bar{x}, \bar{y})(x), D G_{+}(\bar{x}, \bar{z})(x), D H(\bar{x}, 0)(x)\right)$. It follows from the strictly positive homogeneousness of contingent derivatives that, for any $t>0$,

$$
\begin{aligned}
t D F_{+}(\bar{x}, \bar{y})(0) & =D F_{+}(\bar{x}, \bar{y})(t .0)=D F_{+}(\bar{x}, \bar{y})(0), \\
t D G_{+}(\bar{x}, \bar{z})(0) & =D G_{+}(\bar{x}, \bar{z})(t .0)=D G_{+}(\bar{x}, \bar{z})(0), \\
t D H(\bar{x}, 0)(0) & =D H(\bar{x}, 0)(t .0)=D H(\bar{x}, 0)(0) .
\end{aligned}
$$

This, taking to account (3.10) with $x=0$, entails that

$$
t\left(\left\langle c^{*}, y\right\rangle+\left\langle d^{*}, z\right\rangle+\left\langle w^{*}, w\right\rangle\right)+\left\langle d^{*}, \bar{z}\right\rangle \geq 0,
$$

for all $t>0$. Passing to limit as $t \rightarrow 0^{+}$, one has $\left\langle d^{*}, \bar{z}\right\rangle \geq 0$. On the other hand, since $\bar{z} \in-D$, $\left\langle d^{*}, \bar{z}\right\rangle \leq 0$, which in turn implies the equality $\left\langle d^{*}, \bar{z}\right\rangle=0$. Since $c^{*} \in C^{+}, d^{*} \in D^{+}$and $\left\langle d^{*}, \bar{z}\right\rangle=$ 0 , we use (3.10) to deduce that

$$
\left\langle c^{*}, y\right\rangle+\left\langle d^{*}, z\right\rangle+\left\langle w^{*}, w\right\rangle \geq 0,
$$

for all $x \in X, y \in D F_{+}(\bar{x}, \bar{y})(x), z \in D G_{+}(\bar{x}, \bar{z})(x)$ and $w \in D H(\bar{x}, 0)(x)$.

Finally, let (KRZCQ) hold. We will prove that $c^{*} \neq 0$. For every $(z, w) \in Z \times W$, there exist $\beta \geq 0, x \in X, \tilde{z} \in D G_{+}(\bar{x}, \bar{z})(x), \tilde{w} \in D H(\bar{x}, 0)(x)$ and $d \in D$ such that

$$
(z, w)=(\tilde{z}, \tilde{w})+(\beta(d+\bar{z}), 0) .
$$

Reasoning by contraposition, assume that $c^{*}=0$. Then, using (3.11) tells us the inequality $\left\langle d^{*}, \tilde{z}\right\rangle+\left\langle w^{*}, \tilde{w}\right\rangle \geq 0$. From $d \in D, d^{*} \in D^{+}$and $\left\langle d^{*}, \bar{z}\right\rangle=0$, one gets

$$
\left\langle d^{*}, z\right\rangle+\left\langle w^{*}, w\right\rangle=\left\langle d^{*}, \tilde{z}\right\rangle+\left\langle w^{*}, \tilde{w}\right\rangle+\beta\left\langle d^{*}, d\right\rangle+\beta\left\langle d^{*}, \bar{z}\right\rangle \geq 0 .
$$

Since $(z, w) \in Z \times W$ is arbitrary, we arrive at $\left(d^{*}, w^{*}\right)=(0,0)$. This is a contradiction to $\left(c^{*}, d^{*}, w^{*}\right) \neq(0,0,0)$. 


\subsection{Sufficient Optimality Conditions.}

Lemma 3.2. Let $\bar{x} \in \Omega$. If, for each $x_{t} \in \Omega$, there exists $\bar{y}_{t} \in F(\bar{x})$ such that

$$
\left(F\left(x_{t}\right)-\bar{y}_{t}\right) \cap-\operatorname{int} C=\emptyset,
$$

then $\bar{x}$ is a strict minimal solution w.r.t. $\prec_{C}^{l}$ of $(P)$.

Proof. Suppose by contradiction that $\bar{x} \in \Omega$ is not a strict minimal solution w.r.t. $\prec_{C}^{l}$ of $(P)$. Then, there exists $\hat{x} \in \Omega$ such that $F(\hat{x}) \prec_{C}^{l} F(\bar{x})$, i.e., $F(\hat{x})+\operatorname{int} C \supset F(\bar{x})$. Consequently, for all $\bar{y}_{t} \in F(\bar{x})$, there exists $\hat{y}_{t} \in F(\hat{x})$ such that $\bar{y}_{t} \in \hat{y}_{t}+\operatorname{int} C$, or equivalently, $\hat{y}_{t}-\bar{y}_{t} \in-\operatorname{int} C$. Hence, $\left(F(\hat{x})-\bar{y}_{t}\right) \cap-\operatorname{int} C \neq \emptyset$, contradicting to (3.12).

Proposition 3.3. Let $\bar{x} \in X, \bar{z} \in G(\bar{x}) \cap-D, \bar{w}=0 \in H(\bar{x})$ and $F, G, H$ be $C$-convex, $D$-convex, convex, resp. Suppose that there exist $c^{*} \in C^{+} \backslash\{0\}$ and $\left(d^{*}, w^{*}\right) \in D^{+} \times W^{*}$ such that, for all $x \in X$, for all $\bar{y} \in F(\bar{x})$ and for all $(y, z, w) \in\left(D F_{+}(\bar{x}, \bar{y})(x), D G_{+}(\bar{x}, \bar{z})(x), D H(\bar{x}, 0)(x)\right) \backslash$ $\{(0,0,0)\}$,

$$
\left\langle c^{*}, y\right\rangle+\left\langle d^{*}, z\right\rangle+\left\langle w^{*}, w\right\rangle \geq 0,
$$

and $\left\langle d^{*}, \bar{z}\right\rangle=0$. Then, $\bar{x}$ is a strict minimal solution w.r.t. $\prec_{C}^{l}$ of $(P)$.

Proof. Reasoning ad absurdum, assume that $\bar{x}$ is not a strict minimal solution w.r.t. $\prec_{C}^{l}$ of (P). Invoking from Lemma 3.2, there is $x_{t} \in \Omega$ such that, for all $\bar{y}_{t} \in F(\bar{x})$,

$$
\left(F\left(x_{t}\right)-\bar{y}_{t}\right) \cap-\operatorname{int} C \neq \emptyset .
$$

This implies the existence of $x_{t} \in \Omega$ such that, for all $\bar{y}_{t} \in F(\bar{x})$, there exists $y_{t} \in F\left(x_{t}\right)$ satisfying $y_{t}-\bar{y}_{t} \in-\operatorname{int} C$. This is nothing else but there are $y_{t} \in F\left(x_{t}\right), z_{t} \in G\left(x_{t}\right) \cap-D$, and $0 \in H\left(x_{t}\right)$ such that $y_{t}-\bar{y}_{t} \in-\operatorname{int} C$, and thus,

$$
\left\langle c^{*}, y_{t}-\bar{y}_{t}\right\rangle<0
$$

Applying Lemma 2.1 gives us that

$$
\left(y_{t}-\bar{y}_{t}, z_{t}-\bar{z}, 0-\bar{w}\right) \in\left(D F_{+}(\bar{x}, \bar{y})\left(x_{t}-\bar{x}\right), D G_{+}(\bar{x}, \bar{z})\left(x_{t}-\bar{x}\right), D H(\bar{x}, 0)\left(x_{t}-\bar{x}\right)\right) .
$$

Therefore, (3.13) amounts to

$$
\left\langle c^{*}, y_{t}-\bar{y}_{t}\right\rangle+\left\langle d^{*}, z_{t}-\bar{z}\right\rangle=\left\langle c^{*}, y_{t}-\bar{y}_{t}\right\rangle+\left\langle d^{*}, z_{t}-\bar{z}\right\rangle+\left\langle w^{*}, 0\right\rangle \geq 0 .
$$

Because $d^{*} \in D^{+}$and $z_{t} \in G\left(x_{t}\right) \cap-D$, we have $\left\langle d^{*}, z_{t}\right\rangle \leq 0$. This together with $\left\langle d^{*}, \bar{z}\right\rangle=0$ leads that

$$
\left\langle c^{*}, y_{t}-\bar{y}_{t}\right\rangle \geq-\left\langle d^{*}, z_{t}\right\rangle \geq 0
$$

contradicting to (3.14).

Example 3.1. Let $X=\mathbb{R}^{2}, Y=Z=W=\mathbb{R}$ and $C=D=\mathbb{R}_{+}, F\left(x_{1}, x_{2}\right)=\left\{y \in \mathbb{R} \mid y \geq x_{1}^{2}\right\}$, $G\left(x_{1}, x_{2}\right)=\left\{z \in \mathbb{R} \mid z \geq-2 x_{2}\right\}$ and $H\left(x_{1}, x_{2}\right)=\left\{w \in \mathbb{R} \mid w=-x_{2}\right\}$. Then, $\Omega=\left\{\left(x_{1}, x_{2}\right) \in\right.$ $\left.\mathbb{R}^{2} \mid x_{2}=0\right\}$. By taking $\bar{x}=(0,0), \bar{y}=0$ and $\bar{z}=0$, one has $F(x)+\operatorname{int} C \not \supset F(\bar{x}), \forall x \in \Omega$, i.e., $F(x) \nprec_{C}^{l} F(\bar{x}), \forall x \in \Omega$, in other words, $\bar{x}$ is a strict minimal solution w.r.t. $\prec_{C}^{l}$ of $(\mathrm{P})$. We can check that $F$ is $C$-convex, $G$ is $D$-convex, $H$ is convex and $H$ is directionally metrically subregular at $(\bar{x}, 0)$ w.r.t $X$. Since $F_{+}(x)=F(x), G_{+}(x)=G(x)$, it is easy to justify that $F_{+}$is $C$-Aubin at $(\bar{x}, \bar{y}), G_{+}$is $D$-Aubin at $(\bar{x}, \bar{z})$. By some calculations, we get that, for all $x \in X$,

$$
D F_{+}(\bar{x}, \bar{y})(x)=\{y \in \mathbb{R} \mid y \geq 0\}, D G_{+}(\bar{x}, \bar{z})(x)=\left\{z \in \mathbb{R} \mid z \geq-2 x_{2}\right\},
$$




$$
\operatorname{DH}(\bar{x}, 0)(x)=\left\{w \in \mathbb{R} \mid w=-x_{2}\right\} .
$$

Hence,

$$
\left(D G_{+}(\bar{x}, \bar{z}), D H(\bar{x}, 0)\right)(X)+\operatorname{cone}(D+\bar{z}) \times\{0\}=Z \times W,
$$

i.e., (KRZCQ) holds, and thus, all assumptions of Proposition 3.2 are fulfilled. By choosing $\left(c^{*}, d^{*}, w^{*}\right)=(1,0,0)$, one gets that $\left(c^{*}, d^{*}, w^{*}\right) \in\left(\mathbb{R}_{+} \backslash\{0\}\right) \times \mathbb{R}_{+} \times \mathbb{R}$ satisfying $\left\langle d^{*}, \bar{z}\right\rangle=0$ and

$$
\left\langle c^{*}, y\right\rangle+\left\langle d^{*}, z\right\rangle+\left\langle w^{*}, w\right\rangle \geq 0
$$

for every $x \in X, y \in D F_{+}(\bar{x}, \bar{y})(x), z \in D G_{+}(\bar{x}, \bar{z})(x)$ and $w \in D H(\bar{x}, 0)(x)$. In addition, the hypotheses of Proposition 3.3 are also satisfied. So, we can derive from (3.15) that $\bar{x}$ is a strict minimal solution w.r.t. $\prec_{C}^{l}$ of $(\mathrm{P})$.

\section{DUALITY}

In this section, we formulate a Mond-Weir [21] type dual problem and a Wolfe [25] type dual problem of (P) and explore weak and strong duality relations.

4.1. Mond-Weir type duality. For $x^{\prime} \in X, y^{\prime} \in F\left(x^{\prime}\right), z^{\prime} \in G\left(x^{\prime}\right) \cap(-D), w^{\prime} \in H\left(x^{\prime}\right), c^{*} \in$ $C^{+} \backslash\{0\}, d^{*} \in D^{+}$and $w^{*} \in W^{*}$, let us denote

$$
L\left(x^{\prime}, y^{\prime}, z^{\prime}, w^{\prime}, c^{*}, d^{*}, w^{*}\right)=F\left(x^{\prime}\right) .
$$

We consider the Mond-Weir type dual problem of $(\mathrm{P})$ as follows

$$
\left(D_{M W}\right):\left\{\begin{aligned}
& \preceq_{C}^{l}-\max L\left(x^{\prime}, y^{\prime}, z^{\prime}, w^{\prime}, c^{*}, d^{*}, w^{*}\right)=F\left(x^{\prime}\right), \\
& \text { s.t. }\left\langle c^{*}, y\right\rangle+\left\langle d^{*}, z\right\rangle+\left\langle w^{*}, w\right\rangle \geq 0, \\
& \forall(y, z, w) \in \bigcup_{x \in X}\left(D F_{+}\left(x^{\prime}, y^{\prime}\right)(x), D G_{+}\left(x^{\prime}, z^{\prime}\right)(x), D H\left(x^{\prime}, w^{\prime}\right)(x)\right), \\
&\left\langle d^{*}, z^{\prime}\right\rangle+\left\langle w^{*}, w^{\prime}\right\rangle \geq 0, \\
&\left(c^{*}, d^{*}, w^{*}\right) \in\left(C^{+} \backslash\{0\}\right) \times D^{+} \times W^{*} .
\end{aligned}\right.
$$

The feasible set of problem $\left(D_{M W}\right)$ is

$$
\begin{gathered}
\Omega_{M W}:=\left\{\left(x^{\prime}, y^{\prime}, z^{\prime}, w^{\prime}, c^{*}, d^{*}, w^{*}\right) \in X \times Y \times Z \times W \times\left(C^{+} \backslash\{0\}\right) \times D^{+} \times W^{*}, y^{\prime} \in F\left(x^{\prime}\right),\right. \\
z^{\prime} \in G\left(x^{\prime}\right) \cap(-D), w^{\prime} \in H\left(x^{\prime}\right),\left\langle c^{*}, y\right\rangle+\left\langle d^{*}, z\right\rangle+\left\langle w^{*}, w\right\rangle \geq 0, \\
\left.\forall(y, z, w) \in \bigcup_{x \in X}\left(D F_{+}\left(x^{\prime}, y^{\prime}\right)(x), D G_{+}\left(x^{\prime}, z^{\prime}\right)(x), D H\left(x^{\prime}, w^{\prime}\right)(x)\right),\left\langle d^{*}, z^{\prime}\right\rangle+\left\langle w^{*}, w^{\prime}\right\rangle \geq 0\right\} .
\end{gathered}
$$

A point $\left(\overline{x^{\prime}}, \overline{y^{\prime}}, \bar{z}^{\prime}, \bar{w}^{\prime}, \bar{c}^{*}, \bar{d}^{*}, \bar{w}^{*}\right) \in \Omega_{M W}$ is a strict maximal solution w.r.t. $\prec_{C}^{l}$ of $\left(D_{M W}\right)$ if, for all $\left(x^{\prime}, y^{\prime}, z^{\prime}, w^{\prime}, c^{*}, d^{*}, w^{*}\right) \in \Omega_{M W}$,

$$
L\left(\overline{x^{\prime}}, \overline{y^{\prime}}, \overline{z^{\prime}}, \overline{w^{\prime}}, \overline{c^{*}}, \bar{d}^{*}, \bar{w}^{*}\right) \nprec_{C}^{l} L\left(x^{\prime}, y^{\prime}, z^{\prime}, w^{\prime}, c^{*}, d^{*}, w^{*}\right) .
$$

Proposition 4.1. (Weak duality) Suppose that $F, G, H$ be $C$-convex, D-convex, convex, resp. If $x \in \Omega$ and $\left(x^{\prime}, y^{\prime}, z^{\prime}, w^{\prime}, c^{*}, d^{*}, w^{*}\right) \in \Omega_{M W}$, then $F(x) \nprec_{C}^{l} L\left(x^{\prime}, y^{\prime}, z^{\prime}, w^{\prime}, c^{*}, d^{*}, w^{*}\right)$. 
Proof. We assume to the contrary that $F(x) \prec_{C}^{l} L\left(x^{\prime}, y^{\prime}, z^{\prime}, w^{\prime}, c^{*}, d^{*}, w^{*}\right)=F\left(x^{\prime}\right)$. Then, one gets that $F(x)+\operatorname{int} C \supseteq F\left(x^{\prime}\right)$. This ensures the existence of $y \in F(x)$ and $y^{\prime} \in F\left(x^{\prime}\right)$ such that $y-y^{\prime} \in-$ int $C$. Since $c^{*} \in C^{+} \backslash\left\{0_{Y^{*}}\right\}$, one has

$$
\left\langle c^{*}, y-y^{\prime}\right\rangle<0 \text {. }
$$

The fact on $\left(x^{\prime}, y^{\prime}, z^{\prime}, w^{\prime}, c^{*}, d^{*}, w^{*}\right) \in \Omega_{M W}$ ensures that $y^{\prime} \in F\left(x^{\prime}\right), z^{\prime} \in G\left(x^{\prime}\right) \cap(-D), w^{\prime} \in H\left(x^{\prime}\right)$, $\left(c^{*}, d^{*}, w^{*}\right) \in\left(C^{+} \backslash\{0\}\right) \times D^{+} \times W^{*}$,

$$
\left\langle d^{*}, z^{\prime}\right\rangle+\left\langle w^{*}, w^{\prime}\right\rangle \geq 0
$$

and

$$
\left\langle c^{*}, y\right\rangle+\left\langle d^{*}, z\right\rangle+\left\langle w^{*}, w\right\rangle \geq 0, \forall(y, z, w) \in \bigcup_{x \in X}\left(D F_{+}\left(x^{\prime}, y^{\prime}\right)(x), D G_{+}\left(x^{\prime}, z^{\prime}\right)(x), D H\left(x^{\prime}, w^{\prime}\right)(x)\right) .
$$

It follows from the convexity assumptions and Lemma 2.1 that, $\forall x \in X$,

$$
\left(F(x)-y^{\prime}, G(x)-z^{\prime}, H(x)-w^{\prime}\right) \subset\left(D F_{+}\left(x^{\prime}, y^{\prime}\right)\left(x-x^{\prime}\right), D G_{+}\left(x^{\prime}, z^{\prime}\right)\left(x-x^{\prime}\right), D H\left(x^{\prime}, w^{\prime}\right)\left(x-x^{\prime}\right)\right) .
$$

Since $x \in \Omega$, there exists $z \in G(x) \cap(-D)$ and $0 \in H(x)$. This implies that

$$
\left\langle d^{*}, z\right\rangle \leq 0 \text {. }
$$

Furthermore, for any $y \in F(x)$, one has

$$
\begin{aligned}
\left(y-y^{\prime}, z-z^{\prime}, 0-w^{\prime}\right) & \in\left(F(x)-y^{\prime}, G(x)-z^{\prime}, H(x)-w^{\prime}\right) \\
& \subset\left(D F_{+}\left(x^{\prime}, y^{\prime}\right)\left(x-x^{\prime}\right), D G_{+}\left(x^{\prime}, z^{\prime}\right)\left(x-x^{\prime}\right), D H\left(x^{\prime}, w^{\prime}\right)\left(x-x^{\prime}\right)\right),
\end{aligned}
$$

and hence, utilizing (4.3) tells us that

$$
\left\langle c^{*}, y-y^{\prime}\right\rangle+\left\langle d^{*}, z\right\rangle-\left(\left\langle d^{*}, z^{\prime}\right\rangle+\left\langle w^{*}, w^{\prime}\right\rangle\right)=\left\langle c^{*}, y-y^{\prime}\right\rangle+\left\langle d^{*}, z-z^{\prime}\right\rangle+\left\langle w^{*}, 0-w^{\prime}\right\rangle \geq 0 .
$$

Combining this with (4.2) and (4.4), we arrive at

$$
\left\langle c^{*}, y-y^{\prime}\right\rangle \geq-\left\langle d^{*}, z\right\rangle+\left(\left\langle d^{*}, z^{\prime}\right\rangle+\left\langle w^{*}, w^{\prime}\right\rangle\right) \geq 0,
$$

which contradicts with (4.1). This completes the proof.

Proposition 4.2. (Strong duality) Let $\bar{x} \in X, \bar{y} \in F(\bar{x}), \bar{z} \in G(\bar{x}) \cap(-D), \bar{w}=0 \in H(\bar{x})$. Suppose that the following conditions hold:

(i) $\mathrm{WMin}_{C} F(\bar{x})=\{\bar{y}\}, F(\bar{x})$ has the weakly $C$-minimal property,

(ii) $F$, $G, H$ are $C$-convex, $D$-convex, convex, respectively,

(iii) $F_{+}$is $C$-Aubin at $(\bar{x}, \bar{y}), G_{+}$is $D$-Aubin at $(\bar{x}, \bar{z})$,

(iv) $H$ is directionally metrically subregular at $(\bar{x}, 0)$ w.r.t. $X$,

(v) $\left(D G_{+}(\bar{x}, \bar{z}), D H(\bar{x}, 0)\right)(X)+\operatorname{cone}(D+\bar{z}) \times\{0\}=Z \times W$.

If $\bar{x} \in \Omega$ is a strict minimal solution w.r.t. $\prec_{C}^{l}$ of $(P)$, then there exists $\bar{c}^{*} \in C^{+} \backslash\{0\}, \bar{d}^{*} \in D^{+}$ and $\bar{w}^{*} \in W^{*}$ such that $\left(\bar{x}, \bar{y}, \bar{z}, \bar{w}, \bar{c}^{*}, \bar{d}^{*}, \bar{w}^{*}\right)$ is a strict maximal solution w.r.t. $\prec_{C}^{l}$ of $\left(D_{M W}\right)$.

Proof. We derive from Proposition 3.2 the existence of $\left(\overline{c^{*}}, \overline{d^{*}}, \bar{w}^{*}\right) \in C^{+} \backslash\{0\} \times D^{+} \times W^{*}$ such that, for all $x \in X$ and for all $(y, z, w) \in\left(D F_{+}(\bar{x}, \bar{y})(x), D G_{+}(\bar{x}, \bar{z})(x), D H(\bar{x}, 0)(x)\right) \backslash\{(0,0,0)\}$,

$$
\left\langle\bar{c}^{*}, y\right\rangle+\left\langle\bar{d}^{*}, z\right\rangle+\left\langle\bar{w}^{*}, w\right\rangle \geq 0 \text { and }\left\langle\bar{d}^{*}, \bar{z}\right\rangle+\left\langle\bar{w}^{*}, \bar{w}\right\rangle=\left\langle\bar{d}^{*}, \bar{z}\right\rangle=0 .
$$


Hence, $\left(\bar{x}, \bar{y}, \bar{z}, \bar{w}, \bar{c}^{*}, \bar{d}^{*}, \bar{w}^{*}\right) \in \Omega_{M W}$ and $L\left(\bar{x}, \bar{y}, \bar{z}, \bar{w}, \bar{c}^{*}, \bar{d}^{*}, \bar{w}^{*}\right)=F(\bar{x})$. Now, suppose to the contrary that $\left(\bar{x}, \bar{y}, \bar{z}, \bar{w}, \bar{c}^{*}, \bar{d}^{*}, \bar{w}^{*}\right)$ is not a strict minimal solution w.r.t. $\prec_{C}^{l}$ of $\left(D_{M W}\right)$. Then, there exists $\left(x^{\prime}, y^{\prime}, z^{\prime}, w^{\prime}, c^{*}, d^{*}, w^{*}\right) \in \Omega_{M W}$ such that

$$
F(\bar{x})=L\left(\bar{x}, \bar{y}, \bar{z}, \bar{w}, \bar{c}^{*}, \bar{d}^{*}, \bar{w}^{*}\right) \prec_{C}^{l} L\left(x^{\prime}, y^{\prime}, z^{\prime}, w^{\prime}, c^{*}, d^{*}, w^{*}\right),
$$

contradicting with Proposition 4.1. Hence, $\left(\bar{x}, \bar{y}, \bar{z}, \bar{w}, \bar{c}^{*}, \bar{d}^{*}, \bar{w}^{*}\right)$ is a strict maximal solution w.r.t. $\prec_{C}^{l}$ of $\left(D_{M W}\right)$.

Remark 4.1. Let $X=\mathbb{R}^{n}, Y=\mathbb{R}^{p}, Z=\mathbb{R}^{q}, W=\mathbb{R}^{r}, C=\mathbb{R}_{+}^{p}, D=\mathbb{R}_{+}^{q}, F:=\left(f_{1}, \ldots, f_{p}\right), G:=$ $\left(g_{1}, \ldots, g_{q}\right), H:=\left(h_{1}, \ldots, h_{r}\right)$ be single-valued functions, where $f_{i}, i=1, \ldots, p, g_{j}, j=1, \ldots, q$, and $h_{k}, k=1, \ldots, r$, are continuously differentiable functions from $\mathbb{R}^{n}$ to $\mathbb{R}$. Then, for $\bar{x} \in \mathbb{R}^{n}$,

$$
\begin{gathered}
D F_{+}(\bar{x}, f(\bar{x}))(x)=\nabla f(\bar{x})(x)+\mathbb{R}_{+}^{p}, D G_{+}(\bar{x}, g(\bar{x}))(x)=\nabla g(\bar{x})(x)+\mathbb{R}_{+}^{q}, \\
D H(\bar{x}, h(\bar{x}))(x)=\{\nabla h(\bar{x})(x)\}, \forall x \in X .
\end{gathered}
$$

The Mond-Weir dual problem of $(\mathrm{P})$ is

$$
\left(D_{M W}\right):\left\{\begin{array}{c}
\mathbb{R}_{+}^{p}-\max L\left(x^{\prime}, f\left(x^{\prime}\right), g\left(x^{\prime}\right), h\left(x^{\prime}\right), c^{*}, d^{*}, w^{*}\right)=f\left(x^{\prime}\right), \\
\text { s.t. } \nabla f\left(x^{\prime}\right)^{T} c^{*}+\nabla g\left(x^{\prime}\right)^{T} d^{*}+\nabla h\left(x^{\prime}\right)^{T} w^{*}=0, \\
\left\langle d^{*}, g\left(x^{\prime}\right)\right\rangle+\left\langle w^{*}, h\left(x^{\prime}\right)\right\rangle \geq 0 \\
\left(c^{*}, d^{*}, w^{*}\right) \in\left(\mathbb{R}_{+}^{p} \backslash\{0\}\right) \times \mathbb{R}_{+}^{q} \times \mathbb{R}^{r},
\end{array}\right.
$$

since

$$
\begin{aligned}
& \left\langle\nabla f\left(x^{\prime}\right)^{T} c^{*}+\nabla g\left(x^{\prime}\right)^{T} d^{*}+\nabla h\left(x^{\prime}\right)^{T} w^{*}, x\right\rangle \\
& =\left\langle c^{*}, \nabla f\left(x^{\prime}\right)(x)\right\rangle+\left\langle d^{*}, \nabla g\left(x^{\prime}\right)(x)\right\rangle+\left\langle w^{*}, \nabla h\left(x^{\prime}\right)(x)\right\rangle \\
& \geq 0
\end{aligned}
$$

for all $x \in \mathbb{R}^{n}$ if and only if

$$
\nabla f\left(x^{\prime}\right)^{T} c^{*}+\nabla g\left(x^{\prime}\right)^{T} d^{*}+\nabla h\left(x^{\prime}\right)^{T} w^{*}=0 .
$$

When $p=1$, this problem is exactly the Mond-Weir dual problem (see [5, 21]).

Example 4.1. Let $X=\mathbb{R}^{2}, Y=Z=W=\mathbb{R}$ and $C=D=\mathbb{R}_{+}, F\left(x_{1}, x_{2}\right)=\left\{y \in \mathbb{R} \mid y \geq x_{1}^{2}\right\}$, $G\left(x_{1}, x_{2}\right)=\left\{z \in \mathbb{R} \mid z \geq-2 x_{2}\right\}$ and $H\left(x_{1}, x_{2}\right)=\left\{w \in \mathbb{R} \mid w=-x_{2}\right\}$. Consider the following set optimization problem with mixed constraints:

$$
(\mathrm{P}): \preceq_{C}^{l}-\min _{x \in X}\{F(x) \mid G(x) \cap(-D) \neq \emptyset, 0 \in H(x)\} .
$$

Then, $\Omega=\left\{\left(x_{1}, x_{2}\right) \in \mathbb{R}^{2} \mid x_{2}=0\right\}$. For $x^{\prime} \in X, y^{\prime} \in F\left(x^{\prime}\right), z^{\prime} \in G\left(x^{\prime}\right) \cap(-D), w^{\prime} \in H\left(x^{\prime}\right)$, one gets that, for $x \in \mathbb{R}^{2}$,

$$
\begin{gathered}
D F_{+}\left(x^{\prime}, y^{\prime}\right)(x)= \begin{cases}\left\{y \in \mathbb{R} \mid y \geq 2 x_{1}^{\prime} x_{1}\right\}, & \text { if } y^{\prime}=x_{1}^{\prime 2}, \\
\mathbb{R}, & \text { if } y^{\prime}>x_{1}^{\prime 2},\end{cases} \\
D G_{+}\left(x^{\prime}, z^{\prime}\right)(x)= \begin{cases}\left\{z \in \mathbb{R} \mid z \geq-2 x_{2}\right\}, & \text { if } z^{\prime}=-2 x_{2}^{\prime}, \\
\mathbb{R}, & \text { if } z^{\prime}>-2 x_{2}^{\prime},\end{cases} \\
D H\left(x^{\prime}, w^{\prime}\right)(x)=\left\{w \in \mathbb{R} \mid w=-x_{2}\right\} .
\end{gathered}
$$


The Mond-Weir type dual problem of $(\mathrm{P})$ is

$$
\left(D_{M W}\right):\left\{\begin{array}{c}
\preceq_{C}^{l}-\max L\left(x^{\prime}, y^{\prime}, z^{\prime}, w^{\prime}, c^{*}, d^{*}, w^{*}\right)=F\left(x^{\prime}\right), \\
\text { s.t. } c^{*} y+d^{*} z+w^{*} w \geq 0, \\
\forall(y, z, w) \in \bigcup_{x \in \mathbb{R}^{2}}\left(D F_{+}\left(x^{\prime}, y^{\prime}\right)(x), D G_{+}\left(x^{\prime}, z^{\prime}\right)(x), D H\left(x^{\prime}, w^{\prime}\right)(x)\right), \\
d^{*} z^{\prime}+w^{*} w^{\prime} \geq 0, \\
\left(c^{*}, d^{*}, w^{*}\right) \in\left(\mathbb{R}_{+} \backslash\{0\}\right) \times \mathbb{R}_{+} \times \mathbb{R} .
\end{array}\right.
$$

By picking $\bar{x}=(0,0), \bar{y}=0$ and $\bar{z}=0$, we obtain $F(\bar{x})=\{y \in \mathbb{R} \mid y \geq 0\}, G(\bar{x})=\{z \in \mathbb{R} \mid z \geq 0\}$, $H(\bar{x})=\{0\}$, and hence, $\bar{y} \in F(\bar{x}), \bar{z} \in G(\bar{x}) \cap(-D), \bar{w}=0 \in H(\bar{x})$. By some straightforward calculations show that all the assumptions in Proposition 4.2 hold. Taking $\left(c^{*}, d^{*}, w^{*}\right)=(1,0,0)$, we yield the existence of $\left(c^{*}, d^{*}, w^{*}\right) \in\left(\mathbb{R}_{+} \backslash\{0\}\right) \times \mathbb{R}_{+} \times \mathbb{R}$ such that $d^{*} \bar{z}=0$ and

$$
c^{*} y+d^{*} z+w^{*} w \geq 0,
$$

for every $x \in \mathbb{R}^{2}, y \in D F_{+}(\bar{x}, \bar{y})(x), z \in D G_{+}(\bar{x}, \bar{z})(x)$ and $w \in D H(\bar{x}, \bar{w})(x)$. Hence, we derive from Proposition 4.2 that $\left(\bar{x}, \bar{y}, \bar{z}, \bar{w}, \bar{c}^{*}, \bar{d}^{*}, \bar{w}^{*}\right)$ is a strict maximal solution w.r.t. $\prec_{C}^{l}$ of $\left(D_{M W}\right)$.

We can check directly that $\left(\bar{x}, \bar{y}, \bar{z}, \bar{w}, \bar{c}^{*}, \bar{d}^{*}, \bar{w}^{*}\right)$ is a strict maximal solution w.r.t. $\prec_{C}^{l}$ of $\left(D_{M W}\right)$ as follows. Firstly, we will prove that if $\left(x^{\prime}, y^{\prime}, z^{\prime}, w^{\prime}, c^{*}, d^{*}, w^{*}\right) \in \Omega_{M W}$ then $x_{1}^{\prime}=0$. Reasoning by contraposition, assume that there exists $\left(x^{\prime}, y^{\prime}, z^{\prime}, w^{\prime}, c^{*}, d^{*}, w^{*}\right) \in \Omega_{M W}$ and $x_{1}^{\prime} \neq 0$. Since

$$
c^{*} y+d^{*} z+w^{*} w \geq 0,
$$

for all $(y, z, w) \in \bigcup_{x \in \mathbb{R}^{2}}\left(D F_{+}\left(x^{\prime}, y^{\prime}\right)(x), D G_{+}\left(x^{\prime}, z^{\prime}\right)(x), D H\left(x^{\prime}, w^{\prime}\right)(x)\right), 0 \in D G_{+}\left(x^{\prime}, z^{\prime}\right)\left(\left(x_{1}, 0\right)\right)$ and $0 \in D H\left(x^{\prime}, w^{\prime}\right)\left(\left(x_{1}, 0\right)\right)$, one has

$$
c^{*} y \geq 0, \forall y \in \bigcup_{x \in X_{1}} D F_{+}\left(x^{\prime}, y^{\prime}\right)(x)
$$

where $X_{1}=\left\{x \in \mathbb{R}^{2} \mid x_{2}=0\right\}$. Then, there are only two cases as follows.

$*$ Case 1. If $y^{\prime}>x_{1}^{\prime 2}$, then $D F_{+}\left(x^{\prime}, y^{\prime}\right)(x)=\mathbb{R}$ for all $x \in X_{1}$. Hence, we deduce from (4.5) that $c^{*}=0$, contradicting with $\left(x^{\prime}, y^{\prime}, z^{\prime}, w^{\prime}, c^{*}, d^{*}, w^{*}\right) \in \Omega_{M W}$.

$*$ Case 2. If $y^{\prime}=x_{1}^{\prime 2}>0$, then $D F_{+}\left(x^{\prime}, y^{\prime}\right)(x)=\left\{y \in \mathbb{R} \mid y \geq 2 x_{1}^{\prime} x_{1}\right\}$ for all $x \in X_{1}$. Hence, by taking $x=(1,0) \in X_{1}, y=2 x_{1}^{\prime} \in D F_{+}\left(x^{\prime}, y^{\prime}\right)((1,0)), x=(-1,0) \in X_{1}, y=-2 x_{1}^{\prime} \in D F_{+}\left(x^{\prime}, y^{\prime}\right)((-1$, $0)$ ), we deduce from (4.5) that $c^{*}=0$, contradicting with $\left(x^{\prime}, y^{\prime}, z^{\prime}, w^{\prime}, c^{*}, d^{*}, w^{*}\right) \in \Omega_{M W}$.

Thus, if $\left(x^{\prime}, y^{\prime}, z^{\prime}, w^{\prime}, c^{*}, d^{*}, w^{*}\right) \in \Omega_{M W}$ then $x_{1}^{\prime}=0$. Hence, for all $\left(x^{\prime}, y^{\prime}, z^{\prime}, w^{\prime}, c^{*}, d^{*}, w^{*}\right) \in$ $\Omega_{M W}$, one has

$$
F(\bar{x})+\operatorname{int} C \not \supset F\left(x^{\prime}\right) \text {, i.e., } F(\bar{x}) \nprec_{C}^{l} F\left(x^{\prime}\right) .
$$

Equivalently, for all $\left(x^{\prime}, y^{\prime}, z^{\prime}, w^{\prime}, c^{*}, d^{*}, w^{*}\right) \in \Omega_{M W}$,

$$
L\left(\bar{x}, \bar{y}, \bar{z}, \bar{w}, \bar{c}^{*}, \bar{d}^{*}, \bar{w}^{*}\right) \nprec_{C}^{l} L\left(x^{\prime}, y^{\prime}, z^{\prime}, w^{\prime}, c^{*}, d^{*}, w^{*}\right),
$$

i.e., $\left(\bar{x}, \bar{y}, \bar{z}, \bar{w}, \bar{c}^{*}, \bar{d}^{*}, \bar{w}^{*}\right)$ is a strict maximal solution w.r.t. $\prec_{C}^{l}$ of $\left(D_{M W}\right)$. 
4.2. Wolfe type duality. For $x^{\prime} \in X, y^{\prime} \in F\left(x^{\prime}\right), z^{\prime} \in G\left(x^{\prime}\right) \cap(-D), w^{\prime} \in H\left(x^{\prime}\right), c^{*} \in C^{+} \backslash$ $\{0\}, d^{*} \in D^{+}, w^{*} \in W^{*}$, and a fixed point $\tilde{c} \in C \backslash\{0\}$, denote

$$
\widetilde{L}\left(x^{\prime}, y^{\prime}, z^{\prime}, w^{\prime}, c^{*}, d^{*}, w^{*}\right)=F\left(x^{\prime}\right)+\left\langle d^{*}, z^{\prime}\right\rangle \tilde{c}+\left\langle w^{*}, w^{\prime}\right\rangle \tilde{c} .
$$

We define the Wolfe type dual problem of $(\mathrm{P})$ as follows:

$$
\left(D_{W}\right):\left\{\begin{array}{c}
\preceq_{C}^{l}-\max \widetilde{L}\left(x^{\prime}, y^{\prime}, z^{\prime}, w^{\prime}, c^{*}, d^{*}, w^{*}\right)=F\left(x^{\prime}\right)+\left\langle d^{*}, z^{\prime}\right\rangle \tilde{c}+\left\langle w^{*}, w^{\prime}\right\rangle \tilde{c}, \\
\text { s.t. }\left\langle c^{*}, y\right\rangle+\left\langle d^{*}, z\right\rangle+\left\langle w^{*}, w\right\rangle \geq 0, \\
\forall(y, z, w) \in \bigcup_{x \in X}\left(D F_{+}\left(x^{\prime}, y^{\prime}\right)(x), D G_{+}\left(x^{\prime}, z^{\prime}\right)(x), D H\left(x^{\prime}, w^{\prime}\right)(x)\right), \\
\left\langle c^{*}, \tilde{c}\right\rangle=1, \\
\left(c^{*}, d^{*}, w^{*}\right) \in\left(C^{+} \backslash\{0\}\right) \times D^{+} \times W^{*} .
\end{array}\right.
$$

The set of feasible points of $\left(D_{W}\right)$ is

$$
\begin{gathered}
\Omega_{W}:=\left\{\left(x^{\prime}, y^{\prime}, z^{\prime}, w^{\prime}, c^{*}, d^{*}, w^{*}\right) \in X \times Y \times Z \times W \times\left(C^{+} \backslash\{0\}\right) \times D^{+} \times W^{*}, y^{\prime} \in F\left(x^{\prime}\right),\right. \\
z^{\prime} \in G\left(x^{\prime}\right) \cap(-D), 0 \in H\left(x^{\prime}\right),\left\langle c^{*}, y\right\rangle+\left\langle d^{*}, z\right\rangle+\left\langle w^{*}, w\right\rangle \geq 0, \\
\left.\forall(y, z, w) \in \bigcup_{x \in X}\left(D F_{+}\left(x^{\prime}, y^{\prime}\right)(x), D G_{+}\left(x^{\prime}, z^{\prime}\right)(x), D H\left(x^{\prime}, w^{\prime}\right)(x)\right),\left\langle c^{*}, \tilde{c}\right\rangle=1\right\} .
\end{gathered}
$$

A point $\left(\overline{x^{\prime}}, \overline{y^{\prime}}, \overline{z^{\prime}}, \overline{w^{\prime}}, \overline{c^{*}}, \bar{d}^{*}, \bar{w}^{*}\right) \in \Omega_{W}$ is said to be a strict maximal solution w.r.t. $\prec_{C}^{l}$ of $\left(D_{W}\right)$ if, for all $\left(x^{\prime}, y^{\prime}, z^{\prime}, w^{\prime}, c^{*}, d^{*}, w^{*}\right) \in \Omega_{W}$,

$$
\widetilde{L}\left(\overline{x^{\prime}}, \overline{y^{\prime}}, \overline{z^{\prime}}, \bar{w}^{\prime}, \overline{c^{*}}, \bar{d}^{*}, \bar{w}^{*}\right) \nprec_{C}^{l} \widetilde{L}\left(x^{\prime}, y^{\prime}, z^{\prime}, w^{\prime}, c^{*}, d^{*}, w^{*}\right) .
$$

Proposition 4.3. (Weak duality) Suppose that $F, G, H$ be $C$-convex, D-convex, convex, resp. If $x \in \Omega$ and $\left(x^{\prime}, y^{\prime}, z^{\prime}, w^{\prime}, c^{*}, d^{*}, w^{*}\right) \in \Omega_{W}$, then $F(x) \nprec_{C}^{l} \widetilde{L}\left(x^{\prime}, y^{\prime}, z^{\prime}, w^{\prime}, c^{*}, d^{*}, w^{*}\right)$.

Proof. We assume to contrary that

$$
F(x) \prec_{C}^{l} \widetilde{L}\left(x^{\prime}, y^{\prime}, z^{\prime}, w^{\prime}, c^{*}, d^{*}, w^{*}\right)=F\left(x^{\prime}\right)+\left\langle d^{*}, z^{\prime}\right\rangle \tilde{c}+\left\langle w^{*}, w^{\prime}\right\rangle \tilde{c} .
$$

Then, we get that

$$
F(x)+\operatorname{int} C \supseteq F\left(x^{\prime}\right)+\left\langle d^{*}, z^{\prime}\right\rangle \tilde{c}+\left\langle w^{*}, w^{\prime}\right\rangle \tilde{c} .
$$

This implies the existence of $y \in F(x)$ and $y^{\prime} \in F\left(x^{\prime}\right)$ such that

$$
y-\left(y^{\prime}+\left\langle d^{*}, z^{\prime}\right\rangle \tilde{c}+\left\langle w^{*}, w^{\prime}\right\rangle \tilde{c}\right) \in-\operatorname{int} C .
$$

Since $c^{*} \in C^{+} \backslash\{0\}$ and $\left\langle c^{*}, \tilde{c}\right\rangle=1$, we derive from the above inequality that

$$
\left\langle c^{*}, y-y^{\prime}\right\rangle-\left(\left\langle d^{*}, z^{\prime}\right\rangle+\left\langle w^{*}, w^{\prime}\right\rangle\right)=\left\langle c^{*}, y-y^{\prime}\right\rangle-\left(\left\langle d^{*}, z^{\prime}\right\rangle+\left\langle w^{*}, w^{\prime}\right\rangle\right)\left\langle c^{*}, \tilde{c}\right\rangle<0 .
$$

The fact $\left(x^{\prime}, y^{\prime}, z^{\prime}, w^{\prime}, c^{*}, d^{*}, w^{*}\right) \in \Omega_{W}$ justifies that $y^{\prime} \in F\left(x^{\prime}\right), z^{\prime} \in G\left(x^{\prime}\right) \cap(-D), w^{\prime} \in H\left(x^{\prime}\right)$, $\left(c^{*}, d^{*}, w^{*}\right) \in\left(C^{+} \backslash\{0\}\right) \times D^{+} \times W^{*}$ and

$$
\left\langle c^{*}, y\right\rangle+\left\langle d^{*}, z\right\rangle+\left\langle w^{*}, w\right\rangle \geq 0,
$$

for all $(y, z, w) \in \bigcup_{x \in X}\left(D F_{+}\left(x^{\prime}, y^{\prime}\right)(x), D G_{+}\left(x^{\prime}, z^{\prime}\right)(x), D H\left(x^{\prime}, w^{\prime}\right)(x)\right)$. It follows from the convexity assumptions and Lemma 2.1 that, $\forall x \in X$, $\left(F(x)-y^{\prime}, G(x)-z^{\prime}, H(x)-w^{\prime}\right) \subset\left(D F_{+}\left(x^{\prime}, y^{\prime}\right)\left(x-x^{\prime}\right), D G_{+}\left(x^{\prime}, z^{\prime}\right)\left(x-x^{\prime}\right), D H\left(x^{\prime}, w^{\prime}\right)\left(x-x^{\prime}\right)\right)$. 
Since $x \in \Omega$, there exists $z \in G(x) \cap(-D)$ and $0 \in H(x)$. This leads that

$$
\left\langle d^{*}, z\right\rangle \leq 0 \text {. }
$$

Thus, for any $y \in F(x)$, one has

$$
\begin{aligned}
\left(y-y^{\prime}, z-z^{\prime}, 0-w^{\prime}\right) & \in\left(F(x)-y^{\prime}, G(x)-z^{\prime}, H(x)-w^{\prime}\right) \\
& \subset\left(D F_{+}\left(x^{\prime}, y^{\prime}\right)\left(x-x^{\prime}\right), D G_{+}\left(x^{\prime}, z^{\prime}\right)\left(x-x^{\prime}\right), D H\left(x^{\prime}, w^{\prime}\right)\left(x-x^{\prime}\right)\right) .
\end{aligned}
$$

Therefore, we deduce from (4.7) that

$$
\left\langle c^{*}, y-y^{\prime}\right\rangle+\left\langle d^{*}, z\right\rangle-\left(\left\langle d^{*}, z^{\prime}\right\rangle+\left\langle w^{*}, w^{\prime}\right\rangle\right)=\left\langle c^{*}, y-y^{\prime}\right\rangle+\left\langle d^{*}, z-z^{\prime}\right\rangle+\left\langle w^{*}, 0-w^{\prime}\right\rangle \geq 0 .
$$

This along with (4.8) guarantees that

$$
\left\langle c^{*}, y-y^{\prime}\right\rangle-\left(\left\langle d^{*}, z^{\prime}\right\rangle+\left\langle w^{*}, w^{\prime}\right\rangle\right) \geq-\left\langle d^{*}, z\right\rangle \geq 0,
$$

which contradicts with (4.6).

Proposition 4.4. (Strong duality) Let $\bar{x} \in X, \bar{y} \in F(\bar{x}), \bar{z} \in G(\bar{x}) \cap(-D), \bar{w}=0 \in H(\bar{x})$. Suppose that the following conditions hold:

(i) $\mathrm{WMin}_{C} F(\bar{x})=\{\bar{y}\}, F(\bar{x})$ has the weakly $C$-minimal property,

(ii) $F, G, H$ are $C$-convex, D-convex, convex, respectively,

(iii) $F_{+}$is $C$-Aubin at $(\bar{x}, \bar{y}), G_{+}$is D-Aubin at $(\bar{x}, \bar{z})$,

(iv) $H$ is directionally metrically subregular at $(\bar{x}, 0)$ w.r.t. $X$,

(v) $\left(D G_{+}(\bar{x}, \bar{z}), D H(\bar{x}, 0)\right)(X)+\operatorname{cone}(D+\bar{z}) \times\{0\}=Z \times W$.

If $\bar{x} \in \Omega$ is a strict minimal solution w.r.t. $\prec_{C}^{l}$ of $(P)$, then there exists $\bar{c}^{*} \in C^{+} \backslash\{0\}, \bar{d}^{*} \in D^{+}$ and $\bar{w}^{*} \in W^{*}$ such that $\left(\bar{x}, \bar{y}, \bar{z}, \bar{w}, \bar{c}^{*}, \bar{d}^{*}, \bar{w}^{*}\right)$ is a strict maximal solution w.r.t. $\prec_{C}^{l}$ of $\left(D_{W}\right)$.

Proof. According to Proposition 3.2, there exists $\left(\tilde{c^{*}}, \tilde{d^{*}}, \tilde{w^{*}}\right) \in C^{+} \backslash\{0\} \times D^{+} \times W^{*}$ such that, for all $x \in X$ and for all $(y, z, w) \in\left(D F_{+}(\bar{x}, \bar{y})(x), D G_{+}(\bar{x}, \bar{z})(x), D H(\bar{x}, 0)(x)\right) \backslash\{(0,0,0)\}$,

$$
\left\langle\tilde{c}^{*}, y\right\rangle+\left\langle\tilde{d}^{*}, z\right\rangle+\left\langle\tilde{w}^{*}, w\right\rangle \geq 0 \text { and }\left\langle\tilde{d}^{*}, \bar{z}\right\rangle+\left\langle\tilde{w}^{*}, \bar{w}\right\rangle=\left\langle\tilde{d}^{*}, \bar{z}\right\rangle=0 .
$$

Since $\tilde{c} \in C \backslash\{0\}, \tilde{c^{*}} \in C^{+} \backslash\{0\}$, one has $\alpha:=\left\langle\tilde{c^{*}}, \tilde{c}\right\rangle>0$. Setting $\left(\bar{c}^{*}, \bar{d}^{*}, \bar{w}^{*}\right):=\frac{1}{\alpha}\left(\tilde{c^{*}}, \tilde{d}^{*}, \tilde{w}^{*}\right)$, we have

$$
\left(\bar{c}^{*}, \bar{d}^{*}, \bar{w}^{*}\right) \in C^{+} \backslash\{0\} \times D^{+} \times W^{*} \text { and }\left\langle\bar{c}^{*}, \tilde{c}\right\rangle=1 .
$$

Moreover, for all $x \in X$ and for all $(y, z, w) \in\left(D F_{+}(\bar{x}, \bar{y})(x), D G_{+}(\bar{x}, \bar{z})(x), D H(\bar{x}, 0)(x)\right) \backslash\{(0$, $0,0)\}$,

$$
\left\langle\bar{c}^{*}, y\right\rangle+\left\langle\bar{d}^{*}, z\right\rangle+\left\langle\bar{w}^{*}, w\right\rangle=\frac{1}{\alpha}\left(\left\langle\tilde{c}^{*}, y\right\rangle+\left\langle\tilde{d}^{*}, z\right\rangle+\left\langle\tilde{w}^{*}, w\right\rangle\right) \geq 0 .
$$

Hence, $\left(\bar{x}, \bar{y}, \bar{z}, \bar{w}, \bar{c}^{*}, \bar{d}^{*}, \bar{w}^{*}\right) \in \Omega_{W}$. Now, suppose to the contrary that $\left(\bar{x}, \bar{y}, \bar{z}, \bar{w}, \bar{c}^{*}, \bar{d}^{*}, \bar{w}^{*}\right)$ is not a strict maximal solution w.r.t. $\prec_{C}^{l}$ of $\left(D_{W}\right)$. Then, there exists $\left(x^{\prime}, y^{\prime}, z^{\prime}, w^{\prime}, c^{*}, d^{*}, w^{*}\right) \in \Omega_{W}$ such that $\widetilde{L}\left(\bar{x}, \bar{y}, \bar{z}, \bar{w}, \bar{c}^{*}, \bar{d}^{*}, \bar{w}^{*}\right) \prec_{C}^{l} \widetilde{L}\left(x^{\prime}, y^{\prime}, z^{\prime}, w^{\prime}, c^{*}, d^{*}, w^{*}\right)$. Since $\left\langle\bar{w}^{*}, \bar{w}\right\rangle=\left\langle\bar{w}^{*}, 0\right\rangle=0$ and $\left\langle\bar{d}^{*}, \bar{z}\right\rangle=\frac{1}{\alpha}\left\langle\tilde{d}^{*}, \bar{z}\right\rangle=0$, one gets

$$
F(\bar{x})=\widetilde{L}\left(\bar{x}, \bar{y}, \bar{z}, \bar{w}, \bar{c}^{*}, \bar{d}^{*}, \bar{w}^{*}\right) \prec_{C}^{l} \widetilde{L}\left(x^{\prime}, y^{\prime}, z^{\prime}, w^{\prime}, c^{*}, d^{*}, w^{*}\right),
$$

contradicting with Proposition 4.3. So, $\left(\bar{x}, \bar{y}, \bar{z}, \bar{w}, \bar{c}^{*}, \bar{d}^{*}, \bar{w}^{*}\right)$ is a strict maximal solution w.r.t. $\prec_{C}^{l}$ of $\left(D_{W}\right)$. 


\section{Acknowledgements}

The authors would like to thank the Editors for the help in the processing of the article. The authors are grateful to the anonymous referees for the valuable remarks, which helped to improve the paper. The first author is partially supported by Can Tho University.

\section{REFERENCES}

[1] M. Alonso, L. Rodríguez-Marín, Set-relations and optimality conditions in set-valued maps, Nonlinear Anal. 63 (2005), 1167-1179.

[2] N.L.H. Anh, P.Q. Khanh, L.T. Tung, Higher-order radial derivatives and optimality conditions in nonsmooth vector optimization, Nonlinear Anal. 74 (2011), 7365-7379.

[3] N.L.H. Anh, P.Q. Khanh, Higher-order Karush-Kuhn-Tucker optimality conditions for set-valued optimization with nonsolid ordering cones, Positivity 21 (2017), 931-953.

[4] J.P. Aubin, H. Frankowska, Set-Valued Analysis, Birkhäuser, Boston, 1990.

[5] C.R. Bector, M.K. Bector, On various duality theorems in nonlinear programming, J. Optim. Theory Appl. 53 (1987), 509-515.

[6] H.W. Corley, Optimality conditions for maximizations of set-valued functions, J. Optim. Theory Appl. 58 (1988), 1-10.

[7] M. Durea, First and second order optimality conditions for set-valued optimization problems, Rend. Circ. Mat. Palermo 53 (2004), 451-468.

[8] H. Gfrerer, On directional metric regularity, subregularity and optimality conditions for nonsmooth mathematical programs, Set-Valued Var. Anal. 21 (2013), 151-176.

[9] A.H. Hamel, F. Heyde, A. Löhne, B. Rudloff, C. Schrage, Set Optimization And Applications - The State Of The Art: from set relations to set-valued risk measures, Springer Proc. Math. Stat. 151, 2017.

[10] J. Jahn, A.A. Khan, Generalized contingent epiderivative in set-valued optimization: optimality conditions, Numer. Funct. Anal. Optim. 23 (2002), 807-831.

[11] B. Jiménez, V. Novo, Second-order necessary conditions in set constrained differentiable vector optimization, Math. Methods Oper. Res. 58 (2003), 299-317.

[12] A.A. Khan, C. Tammer, C. Zănilescu, Set-Valued Optimization, Springer-Verlag, Berlin, 2016.

[13] P.Q. Khanh, N.M. Tung, Second-order conditions for open-cone minimizers and firm minimizers in setvalued optimization subject to mixed constraints, J. Optim. Theory Appl. 171 (2016), 45-69.

[14] E. Köbis, C. Tammer, J.-C. Yao, Optimality conditions for set-valued optimization problems based on set approach and applications in uncertain optimization, J. Nonlinear Convex Anal. 18 (2017), 1001-1014.

[15] X. Kong, G. Yu, W. Liu, Optimality for set-valued optimization in the sense of vector and set criteria, J. Ineq. Appl. 2017 (2017), 1-11.

[16] D. Kuroiwa, The natural criteria in set-valued optimization, RIMS Kokyuroku 1031 (1998), 85-90.

[17] D. Kuroiwa, On set-valued optimization. Nonlinear Anal. 47 (2001), 1395-1400.

[18] Z. F. Li, G. Y. Chen, Lagrangian multipliers, saddle points, and duality in vector optimization of set-valued maps, J. Math. Anal. Appl. 215 (1997), 297-316.

[19] S.J. Li, K.L. Teo, X.Q. Yang, Higher-order Mond-Weir duality for set-valued optimization, J. Comput. Appl. Math. 217 (2008), 339-349.

[20] D.T. Luc, C. Malivert, Invex optimisation problems, Bull. Aust. Math. Soc. 46 (1992), 47-66.

[21] B. Mond, T. Weir, Generalized concavity and duality. In: Schaible S., Ziemba, W.T. (eds.) Generalized Concavity in Optimization and Economics, Academic Press, New York, 263-279, 1981.

[22] B.S. Mordukhovich, Variational Analysis and Applications, Springer, New York, 2018.

[23] N. Neukel, Order relations of sets and its application in socio-economics, Appl. Math. Sci. 113 (2013), 57115739.

[24] Z. Peng, Z.Wan, Second-order Karush-Kuhn-Tucker optimality conditions for set-valued optimization subject to mixed constraints, Results Math. 73 (2018), 1-20.

[25] P. Wolfe, duality theorem for nonlinear programming. Quart. Appl. Math. 19 (1961), 239-244. 